\title{
A Laban-Based Approach to Emotional Motion Rendering for Human-Robot Interaction
}

\author{
Megumi Masuda, Shohei Kato, and Hidenori Itoh \\ Dept. of Computer Science and Engineering, Graduate School of Engineering, \\ Nagoya Institute of Technology, \\ Gokiso-cho Showa-ku, Nagoya 466-8555, Japan \\ \{masuda, shohey\}@juno.ics.nitech.ac.jp
}

\begin{abstract}
A motion-rendering system that adds target emotion to basic movements of human form robot (HFR) by modifying the movements was created. Pleasure, anger, sadness or relaxation is considered as target emotion. This method not only keeps the user interested, but it also makes the user perceive the robot's emotions and form an attachment to the robot more easily. An experiment was conducted using a real HFR to test how well our system adds target emotion to basic movements. The average of the success rates for adding the target emotion to basic motions were over $60 \%$. This suggests that our method succeeded in adding the target emotions to arbitrary movements.
\end{abstract}

\section{Introduction}

We believe that communication robots will take an active part in our daily lives in the near future. There are many studies about communication robots (e.g., [1], [2]). Moreover, some communication robots are already in use.

Expression of emotions by robots is essential for human-robot communication (HRC). There are two methods for a robot to express an emotional state; one uses verbal information (e.g., 3], 4]), and the other uses nonverbal information (e.g., 5], 6]). In this paper, whole-body expression as nonverbal information is considered as an expression of emotion (e.g., [7, 8]).

Our aim is to create a motion-rendering system that adds target emotion to basic movements by modifying the movements. Canned whole-body movements get old fast because those movements have little variation. The system not only keeps the user interested with much variation, but it also makes the user perceive the robot's emotions and form an attachment to the robot more easily.

First, we introduce Laban movement analysis and Laban's feature value set, which we defined. Next, we describe the method for adding target emotion to arbitrary basic movements, which is based on the result of pilot experiment [9]. In this paper, we consider pleasure, anger, sadness or relaxation as a target emotion. Finally, an experiment is conducted to confirm the usefulness of our method. 
Table 1. Laban's features

\begin{tabular}{|r||rc|c|}
\hline \multicolumn{1}{|c||}{} & & Large & Small \\
\hline \hline Space & the movement directions are & one-sided & different \\
\hline Weight & whole-body movement is & quick & slow \\
\hline Inclination & the whole-body movement is & strong & weak \\
\hline Height & the whole-body is biased & forward & backward \\
\hline Area & the range of whole-body is c large & confined \\
\hline
\end{tabular}

\section{Correlation between Laban's Feature and Emotions Expressed by Movements}

Previously, we proposed a set of motion feature values, called the Laban's feature value set, on the basis of Laban movement analysis (LMA) 9]. In this section, we discuss the features of LMA, and the correlations between robot's motion features and its emotions, estimated by an observer.

\subsection{Laban Movement Analysis}

LMA [10] is a well known theory which developed by Rudolf von Laban, who is widely regarded as a pioneer of European modern dance and a theorist of movement education. It has succeeded Darwin's movement theory [1], which focuses on the structure of an animal's bodily expression. Laban's theory is well suited for science and engineering, because it is mathematical and specific.

\subsection{Laban's Features}

The six main features of LMA are Space, Time, Weight, Inclination, Height and Area. We define Laban's features as follows and Table 1.

- Space represents the bias of whole-body movement.

- Time represents the quickness of whole-body movement.

- Weight represents the powerfulness of whole-body movement.

- Inclination represents the bias for forward of posture.

- Height represents the straightness of posture.

- Area represents the range of whole-body body.

Please refer to [9] for more precise.

\subsection{Correlation between Laban's Features and Expressed Emotions}

We have conducted a pilot experiment to examine the correlation between robot's motion features and the emotions expressed by movements 9. Laban's feature value set is used as the motion feature values. KHR-2HV (Degree-of-freedom = 17, height $=353 \mathrm{~mm}$, shown in Fig. 10 is used as HFR. 
Table 2. Correlations between Laban's Features and Expressed Emotions

\begin{tabular}{|r||c|c|c|c|c|c|}
\hline & Space & Time & Weight & Inclination & Height & Area \\
\hline Pleasure & -0.04 & 0.45 & 0.46 & -0.27 & 0.33 & 0.36 \\
\hline Anger & -0.21 & 0.30 & 0.33 & 0.01 & -0.02 & 0.20 \\
\hline Sadness & 0.03 & -0.38 & -0.42 & 0.47 & -0.51 & -0.39 \\
\hline Relaxation & 0.16 & -0.15 & -0.12 & -0.37 & 0.36 & 0.01 \\
\hline
\end{tabular}

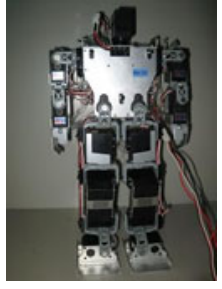

Fig. 1. KHR-2HV

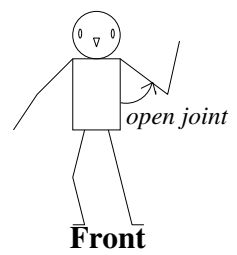

Fig. 2. Link Structure of KHR-2HV and Information

The results of the correlations are in Table 2, Light gray represents a positive correlation (significance level is over 1\%), and dark gray represents a negative correlation (significance level is over 1\%). This table suggests the following.

Pleasure correlates with quickness and powerfulness. It also correlates a bias for backwards posture, straightness of posture, and range of body.

Anger correlates with quickness and powerfulness. It also correlates with movement with moves in different directions, and range of body.

Sadness correlates with slowness and weakness. It also correlates with a bias for forward posture, low posture, and narrowness of body.

Relaxation correlates with slowness and weakness. It also correlates with movement in the same direction, a bias for backwards posture, and straightness of posture.

\section{Method for Adding Emotion to an Arbitrary Basic Movement}

In this section, we describe the method for adding target emotion to arbitrary basic movements. We created a motion rendering system with the consideration that movement can be emotive if it is processed on the basis of the correlation between a robot's motion features and its emotions (Table 20). In this paper, arbitrary basic movements are limited to movements that do not use the feet to reduce the risk of falling.

\subsection{Method for Adding Emotion to Basic Movements}

Our method, whose aim is to add target emotion to basic movements, processes basic movements to change the Laban's feature values on the basis of the 
correlation. In this paper, we adapt HFR KHR-2HV (shown in 1) as the agent. Fig. 2 is a link structure of the KHR-2HV and information about the method.

A basic movement is modified at every unit timepoint $t$ as follows:

Space represents the bias of whole-body movement.

The bias of whole-body movement is related to the movement direction of the extremities and the direction of the face. The system modifies the direction of the face. If the direction of the face is near the average direction of the movement directions of extremities, we consider the directions of movement to be one-sided. The system modifies the direction of the face to add emotion as follows.

$$
\theta_{\text {head }}(t) \leftarrow \theta_{\text {head }}(t)+\left(\theta_{\text {max }}(t)-\theta_{\text {head }}(t)\right) \times e \text { emo }
$$

$\theta_{\text {head }}$ is the angle of the face (neck joint) and $\theta_{\max }$ is the average of all movement directions of extremities. The emo is the correlation coefficient between anger or relaxation and Space.

Time represents the quickness of whole-body movement.

The quickness is related to the angle velocities. The system modifies movement to be quicker. The system shortens the time necessary to rotate a certain angle to add emotion as follows.

$$
\text { time_interval }(t) \leftarrow \text { time_interval }(t)(1-a)
$$

The time_interval is the time necessary to rotate a certain angle. The $a$ is the weight coefficient.

$\boldsymbol{W}$ eight represents the powerfulness of whole-body movement.

Powerfulness is related to angle accelerations. Powerfulness changes at linearly with Time,

$$
\ddot{\theta}(t) \leftarrow \frac{\dot{\theta}(t+1)}{(1-a)}-\frac{\dot{\theta}(t)}{(1-a)}=\frac{(\dot{\theta}(t+1)-\dot{\theta}(t))}{(1-a)}=\frac{\ddot{\theta}(t)}{(1-a)}
$$

Inclination represents the bias for forward posture.

The bias for forward posture is related to the center gravity of the body. The system makes the object bend forward by changing the angle of the waist The system modifies the angle of the waist to add emotion as follows.

$$
\theta_{\text {waist }}(t) \leftarrow \theta_{\text {waist }}(t)+b \times e m o
$$

$\theta_{\text {waist }}$ is the angle of the waist (waist joint). The emo is the correlation coefficient between pleasure, sadness, or relaxation and Inclination. The $b$ is the weight coefficient.

Height represents the straightness of posture.

The straightness of the posture is related to the center gravity of the body. The system raises both hands of the KHR-2HV. The system modifies the angle of the shoulder joint to add emotion as follows.

$$
\theta_{\text {shoulder }}(t) \leftarrow \theta_{\text {shoulder }}(t)+c \times e m o
$$

$\theta_{\text {shoulder }}$ is the angle of the shoulder (screw joint). The emo is the correlation coefficient between pleasure, sadness, or relaxation and Height. 
Area represents the range of the body.

The range of the body is related to the quadrilateral area, which is made by the four extremity points of the extremities, on the horizontal plane. The system makes both hands nearly horizontal. The system modifies the angle of the shoulder joint to add emotion as follows.

$\theta_{\text {shoulder } 2}(t) \leftarrow \theta_{\text {shoulder } 2}(t)-\left(180-\theta_{\text {shoulder } 2}(t)\right)$ emo(when $\theta_{\text {shoulder } 2}(t)$ is over 90$)$ (6) $\theta_{\text {shoulder } 2}(t) \leftarrow \theta_{\text {shoulder } 2}(t)+\theta_{\text {shoulder } 2}(t) \times$ emo $\quad$ (when $\theta_{\text {shoulder } 2}(t)$ is under 90$)$ ( 7 )

$\theta_{\text {shoulder } 2}$ is the angle of the shoulder (open joint). $\theta_{\text {shoulder } 2}$ is 90 when the arm turns to horizontal. The emo is the correlation coefficient between pleasure, anger, and sadness and Area.

\section{Impression Assessment}

We conduct an experiment to test the usefulness of our method. It is the experiment to see if people could identify the emotions we attempted to add to the robot's movements. There were twenty-one subjects between the ages of 20 and 40. They observed KHR-2HV's whole-body movements for about eight seconds and estimated its emotions. Three basic movements (shown in Fig. 3) were prepared. There were four processed movements for the each of the basic movements. We explained the experimental procedure to the subjects and conducted the experiment. The questionnaires used for the experiment are shown in Figs. 4 and 5. The procedure of the experiment is as follows.

1. Subjects watch the basic movement of KHR-2HV. The subjects estimate and mark how strongly they think each emotion is expressed through the basic movement. They marked the segment in the questionnaire in Fig. 4 to answer. The more strongly the subject perceives that the robot expresses the target emotion, the closer the mark should be to 1 . The less emotion the subject perceives, the closer the mark should be to 0 . Homogeneous transformation following the marking was done to quantify the estimation between 0 to 100. The averages of certain emotion's quantified estimations are called the "average of subject's estimations".

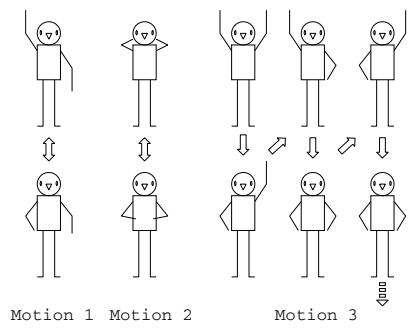

Fig. 3. Beckoning Motions

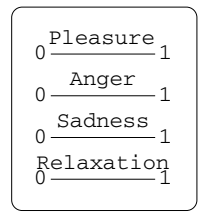

Fig. 4. Questionnaire for the basic movements

Pleasure| Anger | Sadness |Relaxation| None

Fig. 5. Questionnaire for additional emotions 


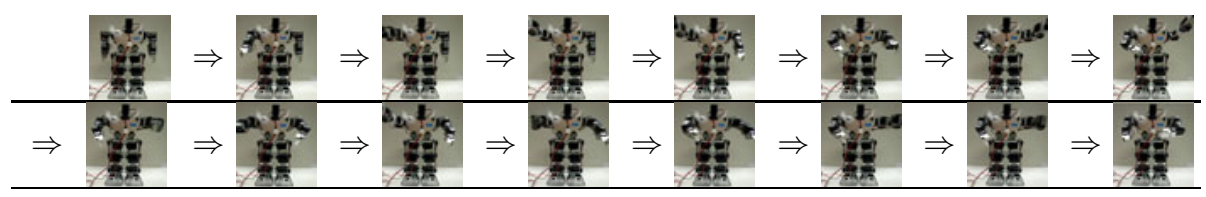

Fig. 6. An Example of Basic Movement (Motion 3)

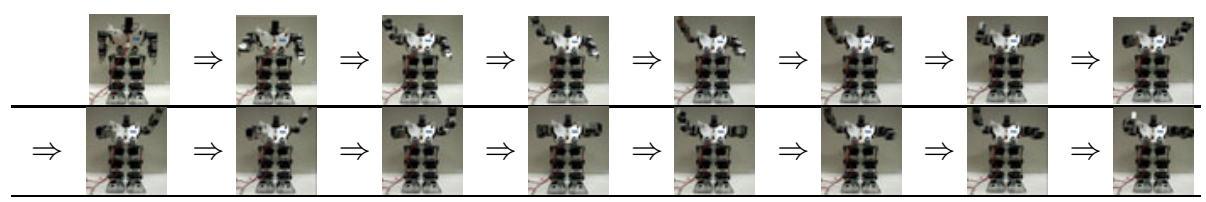

Fig. 7. An Example of Processed Movement (target emotion : Relaxation)

2. The subjects watch a processed movement. The subject evaluates what emotion is added. The evaluations are expressed by marking at the right point of Fig. 5 .

3. The subjects watch the basic movement again.

4. Two and 3 are repeated until four processed movements run out.

We present any movement again if the subject requests it.

The above experiment was conducted three times with different basic movements. Because our aim is to add emotion to arbitrary movements, the three basic movements were made as movements giving different impressions. Fig. 3 shows these motions. For example, continuous snapshots of the processed movement of Motion 3 (shown in Fig. 6) is shown in Fig. 7.

\section{The Rate of Success}

The averages of subjects' estimations are shown in Table 3. The averages of subjects' estimations are between 0 and 100; bigger values mean the movement expresses the emotion more strongly. Table 3 suggests that the three basic movements give different impressions.

\subsection{Rates of Success for Adding the Target Emotion}

The rates of emotion felt by subjects, which is the rate of the subjects feeling certain emotion at certain processed movement, are shown in Table 4 This shows that intended emotions are best supported by the subjects.

The rates of success of adding emotion are shown in Table 5. These are the rate of the subjects identifying the targeted additional emotion.

First, we explain the rates of success of adding the target emotion for each emotion. All emotions had a high rate of success. The rate of success for adding sadness was especially high. Meanwhile the rate of success for adding sadness 
Table 3. Averages of Subjects' Estimations of Basic Movements

\begin{tabular}{|r||c|c|c|}
\hline & Motion1 & Motion2 & Motion3 \\
\hline \hline Pleasure & 39 & 16 & 68 \\
\hline Anger & 30 & 13 & 30 \\
\hline Sadness & 15 & 27 & 6 \\
\hline Relaxation & 15 & 27 & 14 \\
\hline
\end{tabular}

Table 4. Rates of emotion felt by subjects

\begin{tabular}{|r||c|c|c|c|}
\hline target $\backslash$ felt & Pleasure & Anger & Sadness & Relaxation \\
\hline \hline Pleasure & 58.7 & 29.4 & 1.6 & 4.8 \\
\hline Anger & 34.9 & 49.2 & 1.6 & 2.4 \\
\hline Sadness & 0.8 & 8.7 & 82.5 & 4.8 \\
\hline Relaxation & 8.7 & 7.1 & 16.7 & 59.5 \\
\hline
\end{tabular}

Table 5. Rates of Success of Adding Emotion

\begin{tabular}{|r||c|c|c||c|}
\hline & Motion1 & Motion2 & Motion3 & Average \\
\hline \hline Pleasure & 64.3 & 54.8 & 57.1 & 58.7 \\
\hline Anger & 73.8 & 31.0 & 42.9 & 49.2 \\
\hline Sadness & 85.7 & 88.1 & 73.8 & 82.5 \\
\hline Relaxation & 50.0 & 71.4 & 57.1 & 59.5 \\
\hline \hline Average & 68.5 & 61.3 & 57.7 & 62.5 \\
\hline
\end{tabular}

to Motion 3 is comparatively low. We think the reason is that the strength of pleasure for Motion 3 is high (see Table 3). In this particular example, it is difficult to add sadness to Motion 3, because the basic movement expresses strong pleasure; the opposite of sadness in Russell's circumplex model [12. The rate of success for adding anger was comparatively low. We think the reason is that there were few anger movements in pilot experiments. Therefore, it is probable that the motion features of anger were not distilled sufficiently.

Next, we explain the rates of success for adding the target emotion to each basic movement. All basic movements had high rates of success. The rate of success for adding the target emotion to Motion 3 is comparatively low. We think the reason is the lowness of the rate of success for adding sadness.

\section{Related Works}

There are several studies about distilling Laban's features. The robot used by Nakata et al. 13 had three joints and moves on wheels. In contrast, we proposed the method for distilling a Laban's feature value set on a robot that is an HFR with much more joints. An HFR is the type of robot better adapted to mental-like interaction. Maeda et al. 7] studied emotions detection from body movements. They used images of humans and robots. Their information retrieved from the images are comparatively less. In contrast, our Laban's feature value set is sensitive to whole-body movements. Moreover, we considered body movements by a real robot, KHR-2HV. It is natural from a lot of studies (e.g., [14, [15]) that a robot agent can create more positive impressions than a virtual agent.

There are some studies of selecting behavior suitable for situation (e.g., [16], [17]). Our system will make robot more natural by joining hands with these studies. Amaya et al. 18] introduced a model to generate emotional animation 
from neutral human motion. They deal with two emotions and two motion features In contrast, we deal with four emotions and six motion features based on Laban movement analysis.

\section{Conclusion}

We proposed the set of motion feature values, called the Laban's feature value set, on the basis of Laban movement analysis (LMA). We described a concrete method of modifying arbitrary basic movements to add emotions for real human form robot. We conducted an experiment to test if our motion rendering system could add target emotion to basic movements. The results suggest that our method added the target emotion to the movements.

There are some challenges. Our next aim is adding emotion at the intended strength. In this experiment, we used only three basic movements. In the future, we will dedicate to experiment with much more movements to confirm the usefulness of our method. Moreover, our final aim is to build a system that can create its own effectual movements automatically.

\section{Acknowledgment}

This work was supported in part by the Ministry of Education, Science, Sports and Culture, Grant-in-Aid for Scientific Research under grant \#20700199.

\section{References}

1. Bennewitz, M., Faber, F., Joho, D., Behnke, S.: Fritz - A Humanoid Communication Robot. In: Proceedings of the IEEE International Symposium on Robot and Human Interactive Communication (RO-MAN), pp. 1072-1077 (2007)

2. Mitsunaga, N., Miyashita, T., Ishiguro, H., Kogure, K., Hagita, N.: Robovie-IV: A Communication Robot Interacting with People Daily in an Office. In: IROS, pp. 5066-5072. IEEE, Los Alamitos (2006)

3. Breazeal, C.: Emotion and Sociable Humanoid Robots. International Journal of Human-Computer Studies 59, 119-155 (2002)

4. Hara, I., Asano, F., Asoh, H., Ogata, J., Ichimura, N., Kawai, Y., Kanehiro, F., Hirukawa, H., Yamamoto, K.: Robust Speech Interface Based on Audio and Video Information Fusion for Humanoid HRP-2. In: IEEE/RSJ International Conference on Intelligent Robots and Systems (IROS 2004), pp. 2404-2410 (2004)

5. Brooks, A.G., Arkin, R.C.: Behavioral overlays for non-verbal communication expression on a humanoid robot. Autonomous Robots 22, 55-74 (2007)

6. Itoh, C., Kato, S., Itoh, H.: A Characterization of Sensitivity Communication Robots Based on Mood Transition. In: Ho, T.-B., Zhou, Z.-H. (eds.) PRICAI 2008. LNCS (LNAI), vol. 5351, pp. 959-964. Springer, Heidelberg (2008)

7. Maeda, Y., Tanabe, N.: Basic Study on Interactive Emotional Communication by Pet-type Robot. Transactions of the Society of Instrument and Control Engineers 42, 359-366 (2006) (in Japanese) 
8. Hattori, M., Nakabo, Y., Tadokoro, S., Takamori, T., Yamada, K.: An analysis of the Bunraku puppet's motions based on the phase correspondence of the puppet's motions axis-for the generation of humanoid robots motions with fertile emotions. In: IEEE International Conference on Systems, Man, and Cybernetics, pp. 10411046 (1999)

9. Masuda, M., Kato, S., Itoh, H.: Emotion Detection from Body Motion of Human Form Robot Based on Laban Movement Analysis. In: Yang, J.-J., Yokoo, M., Ito, T., Jin, Z., Scerri, P. (eds.) PRIMA 2009. LNCS, vol. 5925, pp. 322-334. Springer, Heidelberg (2009)

10. Laban, R.V.: Mastery of Movement. Princeton Book Co. Pub. (1988)

11. Darwin, C.: On the Expression of the Emotions in Man and Animals. John Murray $(1872)$

12. Russell, J.A.: A circumplex model of affect. Journal of Personality and Social Psychology, 1161-1178 (1980)

13. Nakata, T., Mori, T., Sato, T.: Analysis of Impression of Robot Bodily Expression. Journal of Robotics and Mechatronics 14, 27-36 (2002)

14. Wainer, J., Feil-Seifer, D.J., Shell, D.A., Mataric, M.J.: Embodiment and HumanRobot Interaction. In: 16th IEEE International Conference on Robot \& Human Interactive Communication, pp. 872-877 (2007)

15. Powers, A., Kiesler, S., Fussell, S., Torrey, C.: Comparing a computer agent with a humanoid robot. In: 2nd ACM/IEEE International Conference on Human-Robot Interaction (HRI 2007), pp. 145-152 (2007)

16. Sawada, T., Takagi, T., Fujita, M.: Behavior selection and motion modulation in emotionally grounded architecture for QRIO SDR-4X II. In: Proceedings of the IEEE International Conference on Intelligent Robots and Systems, vol. 3, pp. $2514-2519(2004)$

17. Kim, Y., Kim, Y., Kim, J.: Behavior selection and learning for synthetic character. In: Proc. of the IEEE Congress on Evolutionary Computation, pp. 898-903 (2004)

18. Amaya, K., Bruderlin, A., Calvert, T.: Emotion from motion. In: Proceedings of the conference on Graphics interface 1996, pp. 222-229. Canadian Information Processing Society (1996) 